\title{
Intestinal microbiota in nephrotic children treated with immunosuppressive agents
}

\author{
Robert Szlachciński ${ }^{1}$, Aleksandra Szlachcińska² , Łukasz Szlachciński ${ }^{3}$, Iwona Borycz-Stevens ${ }^{4}$, \\ Feras Almeer ${ }^{5}$, Marcin Tkaczyk ${ }^{4,6}$ \\ 'Department of Neonatology, Tomaszow Health Center, Tomaszow Mazowiecki, Poland \\ 2Department of Thoracic Surgery and Respiratory Rehabilitation, Medical University of Lodz, Copernicus Memorial Hospital, \\ Lodz, Poland \\ ${ }^{3}$ Department of Anesthesiology and Intensive Therapy, Polish Mother's Memorial Hospital Research Institute, Lodz, Poland \\ ${ }^{4}$ Department of Paediatrics, Immunology and Nephrology, Polish Mother's Memorial Hospital Research Institute, Lodz, Poland \\ ${ }^{5}$ Department of Obstetrics, Perinatology and Gynecology, Polish Mother's Memorial Hospital Research Institute, Lodz, Poland \\ ${ }^{6}$ Division of Didactics in Pediatrics, Medical University of Lodz, Lodz, Poland
}

\section{ABSTRACT}

\begin{abstract}
Aim of the study: The analysis of the effect of immunosuppressive therapy on gut microbiota in pediatric patients with stable idiopathic nephrotic syndrome (INS) treated with different protocols of immunosuppressive therapy and examined the changes in their gut microbiota components along with accompanying clinical symptoms.

Material and methods: The study consisted of 44 children with INS divided into 3 groups according to treatment protocols (group A: 18 children on cyclosporine A [CsA], 9 of which receiving additional glucocorticosteroids [GCS]; group B: 17 children on GCS; and group C: 9 children on cyclophosphamide [CYC] and GCS), along with 20 healthy children serving as controls. Intestinal microflora was analysed with microbiological diagnostics based on KyberStatus and KyberMyk tests. Additional laboratory blood and urine tests were performed along with history data and clinical symptoms analysis. Study was approved by local Ethical Committee. All caregivers gave an informed consent for participation.

Results: Total number of bacterial colonies was significantly lower in group A $(p<0.001)$ and group B children $(p=0.04)$ when compared to the healthy controls. Group C children had a significantly lower number of Bifidobacterium colonies than the controls $(p=0.01)$, while number of Candida colonies were significantly higher in group A subjects than in controls $(p=0.01)$. No significant correlation between clinical symptoms reported by patients and the therapy used was found. Degree of disbiosis did was not related to the patients' complaints either.

Conclusions: Immunosuppressed INS paediatric patients who are in remission had significantly unfavourable changes in their intestinal microbiota. Patient on chronic CsA therapy showed higher degree of dysbiosis.
\end{abstract}

\section{KEY WORDS:}

dysbiosis, idiopathic nephrotic syndrome, gut microbiota, immunosuppressive therapy, microbiological diagnostic.

\section{ADDRESS FOR CORRESPONDENCE:}

Marcin Tkaczyk, Department of Paediatrics, Immunology and Nephrology, Polish Mother's Memorial Hospital Research Institute, 281/289 Rzgowska St., 93-338 Lodz, Poland, ORCID: 0000-0003-1753-7560, e-mail: mtkaczyk@uni.lodz.pl 


\section{INTRODUCTION}

Animal models show that immunosuppressive treatment can lead to microfloral disturbances or intestinal dysbiosis [1]. The potentially harmful effect of long-term dysbiosis on the human organism is still under debate. Some authors postulate that the detrimental action is associated with the loss of the protective function of probiotics, an increase in metabolic toxins, digestion and absorption impairment, and immune system disturbances $[2,3]$. On the other hand, it has been proven that a properly functioning immune system maintains the intestinal microflora in physiological equilibrium [4]. We know from human and animal studies that the physiological microflora protects the intestinal ecosystem from pathological colonization and carcinogenesis [5]. It works closely with gut associated lymphoid tissue (GALT) as an important component of the human immune system. It affects metabolic functions related to nutrition, immunological function and intestinal barrier protection. The protective bacteria reduce oxidative stress and affect the maturation of endothelium by producing short chain fatty acids, vitamins $B$ and $K$, and butyric acid [6]. The acidophilic bacteria maintain the $\mathrm{pH}$ of the small intestine and regulates gastrointestinal motility by synthesizing anti-diarrheal postbiotics (acetic acid, propionic acid, and butyric acid) [6]. Intestinal bacteria are involved in digestion, absorption of electrolytes and trace elements, metabolism of bile acids, lipid metabolism, and metabolism of nitrogen compounds. Furthermore, microbiota regulates angiogenesis and the development of intestinal microcirculation.

The clinical significance of microbiota imbalance was described in patients with bowel disease (enterocolitis, irritable bowel syndrome, inflammatory bowel disease, and pseudomembranous colitis) and systemic disorders (atopic dermatitis, rheumatoid arthritis, and fungal infections), as well as primary and acquired immunodeficiency (after radiotherapy and chemotherapy or organ transplantation). These imbalances were related not only to the disease itself, but also to the immunosuppressive treatment. Intestinal dysbiosis could be a source of selected clinical symptoms observed in immunocompromised patients and may influence the course of the disease. Paediatric relapsing idiopathic nephrotic syndrome (INS) patients show gut microbiota dysbiosis, characterized by a decreased proportion of butyric acid-producing bacteria and lower faecal butyric acid quantities, concomitant with reduced circulatory regulatory $\mathrm{T}$ cells (Tregs) [7].

In order to broaden the clinical knowledge concerning this issue, and to study the impact of immunosuppressive therapy on gut microbiota, we conducted a cross-sectional study on children with INS treated with different protocols of immunosuppressants.

\section{MATERIAL AND METHODS}

\section{PATIENT RECRUITMENT AND ETHICAL CONSIDERATIONS}

The study was designed as a cross-sectional clinical analysis of the intestinal microbiota in 44 individuals with INS on immunosuppressive therapy with a stable clinical course of the disease along with 20 healthy individuals all aged 2-18 years. The diagnosis of INS was based on criteria of International Study of Kidney Disease in Children [8]. This study was approved by the Ethics Committee at the Polish Mother's Memorial Hospital Research Institute. Written informed consents were obtained from parents of all participants. The study comprised faecal sample analysis from all 64 participants after dividing them into 4 groups (Table 1). Representing group A were 18 children with INS (7 females, 11 males; aged 2-14 years) treated with oral cyclosporine A (CsA) at a mean dose of $3.7 \mathrm{mg} /$ $\mathrm{kg} / 24 \mathrm{~h}$ given in two doses; 9 of the 18 children received prednisone (GCS) at a fixed dose ranging $10-15 \mathrm{mg} /$ $\mathrm{m}^{2} / 48 \mathrm{~h}$. Group B consisted of 17 children with INS ( 8 females, 9 males; aged $2-17$ years) treated with oral prednisone at a dose not exceeding $40 \mathrm{mg} / \mathrm{m}^{2} / 48 \mathrm{~h}$. In group C, 9 children (3 females, 6 males; aged 3-12 years) were treated with cyclophosphamide (CYC) at a dose of $2.5 \mathrm{mg} / \mathrm{kg} / 24 \mathrm{~h}$. All 9 children in group C received prednisone at a dose of $30-40 \mathrm{mg} / \mathrm{m}^{2} / 48 \mathrm{~h}$. Finally, 20 healthy children (7 females, 13 males; aged 2-15 years) served as controls in group D.

The primary inclusion criterion was INS with immunosuppressive therapy with a stable clinical course of the disease (groups A, B, and C). Exclusion criteria included exacerbation of INS; antibiotic or probiotic usage within two months prior to the study; chronic or acute intestinal disease affecting the intestinal flora. Clinical data and history were collected by a specially tailored questionnaire filled out for each patient at the time of the study, and also 6 months after the study for group A individuals. The questionnaire described the INS course with a special regard to the relapses and treatment, other co-morbidities, antibiotic and probiotic intake, as well as gastrointestinal and neurological symptoms and skin involvement. The severity of symptoms was assessed by children and/or their caregivers. For group A, further blood and urine samples were also collected, with specific consideration

TABLE 1. Description of the study groups and healthy controls

\begin{tabular}{|l|c|c|c|c|}
\hline Characteristic & Group A & Group B & Group C & $\begin{array}{c}\text { Group D } \\
\text { (control) }\end{array}$ \\
\hline $\begin{array}{l}\text { Number } \\
\text { of participants }\end{array}$ & 18 & 17 & 9 & $\begin{array}{c}\text { 20 healthy } \\
\text { participants }\end{array}$ \\
\hline Treatment & CSA + GCS & GCS & CYC + GCS & - \\
\hline
\end{tabular}

CSA - cyklosporine A, GCS - glicocorticosteroids, CYC - cyclophosphamide 
for extended cholesterol, triglycerides, and albumin concentration testing.

\section{COLLECTION OF STOOL SAMPLES AND DATA ANALYSIS}

The intestinal microflora from the subjects was analysed with microbiological diagnostics based on KyberStatus and KyberMyk tests, which are developed by the Institute for Microecology (Herborn, Germany) [9]. These methods provide qualitative and quantitative description of intestinal bacterial and fungal species. Both tests assessed colony forming units (CFU) of microbiota per 1 gram of biological material. The material consisted of faecal samples, which were transported in special containers provided by the Institute of Microecology, 60-190 Poznań, 10 Sielska St. Samples were sent to the laboratory immediately after collection. Delivery time did not exceed 3 days.

Collection of material was done from 8 different places after homogenizing the faecal sample collecting $0.25 \mathrm{~g}$ of feces for testing. The material collected was then placed in a 2,250 $\mathrm{ml}$ of sterile saline (10:1 dilution). The solution was vortexed and serially diluted with successive test tubes, obtaining a $10: 8$ series dilution.

For the KyberStatus test $50 \mu \mathrm{l}$ of each dilution were plated onto enriched or selective agar media. Viable bacterial cell counts in feces were enumerated on the following media: 5\% sheep blood agar (BioMerieux) for total bacterial count, Schaedler agar (Heipha) for anaerobic Bacteroides, DIC agar (Heipha) for Bifidobacteria, CPS ${ }^{\circledR}$ (BioMerieux) for Enterococcus, Escherichia coli, Enterobacteriaceae spp., and Pseudomonas spp., Endo's medium (Heipha) for E. coli Biovare, SPM ${ }^{\circledast}$ (Heipha) for anaerobic Clostridium, and Rogosa medium with peroxidase and TMB (Heipha) for detecting Lactobacilli, and determining the proportion producing $\mathrm{H}_{2} \mathrm{O}_{2}$.

The cultures were grown under appropriate conditions; the plates were incubated under aerobic $(24 \mathrm{~h})$ or anoxic $(48 \mathrm{~h})$ conditions at $37^{\circ} \mathrm{C}$.

In order to determine the presence and quantify molds and Candida spp. in the faecal samples, Kyber-

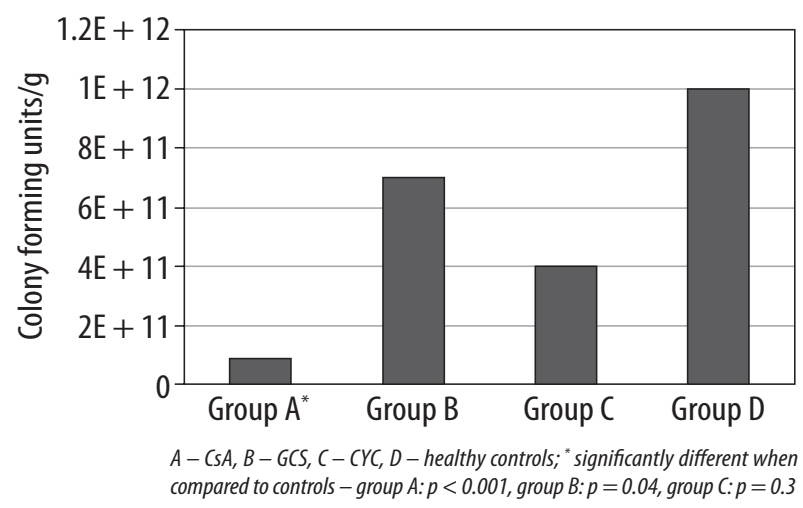

FIGURE 1. Maximum number of bacterial growth in the study groups
Myk test was carried out. 0.25 gram samples of feces were diluted in $2.5 \mathrm{ml}$ of Trypsin-EDTA with $25 \mu \mathrm{l}$ penicillin/streptomycin to inhibit bacterial growth. After homogenization, the solution was placed for 15 minutes in the incubator at $37^{\circ} \mathrm{C}$. Then, $400 \mu \mathrm{l}$ aliquot was transferred to $1.6 \mathrm{ml} \mathrm{PBS}$ for rinsing. Two separate volumes of $100 \mu \mathrm{l}$ each were transferred from the PBS solution into 2 separate Sabouraud agar media with chloramphenicol (BioMerieux). Both plates were incubated for $48 \mathrm{~h}$; one at $37^{\circ} \mathrm{C}$ for $48 \mathrm{~h}$, while the other at room temperature. This allowed to distinguish mushrooms from pathologic fungi. Identification of Candida was done using the chromogenic CHROMagar Candida media (Becton Dickinson).

Additional laboratory tests for children treated with CsA included FBC, kidney function tests, and urinalysis. The CsA concentrations in blood were determined by EMIT (Synevo).

\section{DATA ANALYSIS}

The main parameters analysed were the differences in the quantities of particular strains of bacteria and fungi. Results obtained from each group were compared with other groups' results, including the control. Data obtained from the questionnaire was also analysed. Shapiro-Wilk test was used to assess the normality of distribution of variables. The median and 25-75 interquartiles defined the qualitative variables. Mann-Whitney test, Yates-corrected $\chi^{2}$ test, Fisher's exact test, and Kruskal-Wallis test were used to evaluate differences between groups. Correlation was assessed by Spearman rank correlation coefficient. A $p$-value $<0.05$ was considered significant. Calculations were done using STATISTICA 7.0 software.

\section{RESULTS}

\section{MICROBIOTA}

\section{Total number of bacterial colonies}

The total number of bacterial colonies was lowest in children treated with CsA and significantly different when compared with the healthy controls $(p<0.001)$. Children treated only with GCS also had a significantly lower number of colonies ( $p=0.04$ ), while the CYC-treated children did not show a significant difference when compared with the controls $(p=0.3)$. The significance of this data was confirmed by Mann-Whitney test $(p=0.05)$ as shown in Figure 1.

When individual study groups $\mathrm{A}, \mathrm{B}$, and $\mathrm{C}$ were compared with each other, the total number of bacterial colonies was significantly lower in group A - CsA in comparison to group B - GCS $(p=0.007)$, as well as when compared with group C - CYC $(p=0.04)$. There was no 


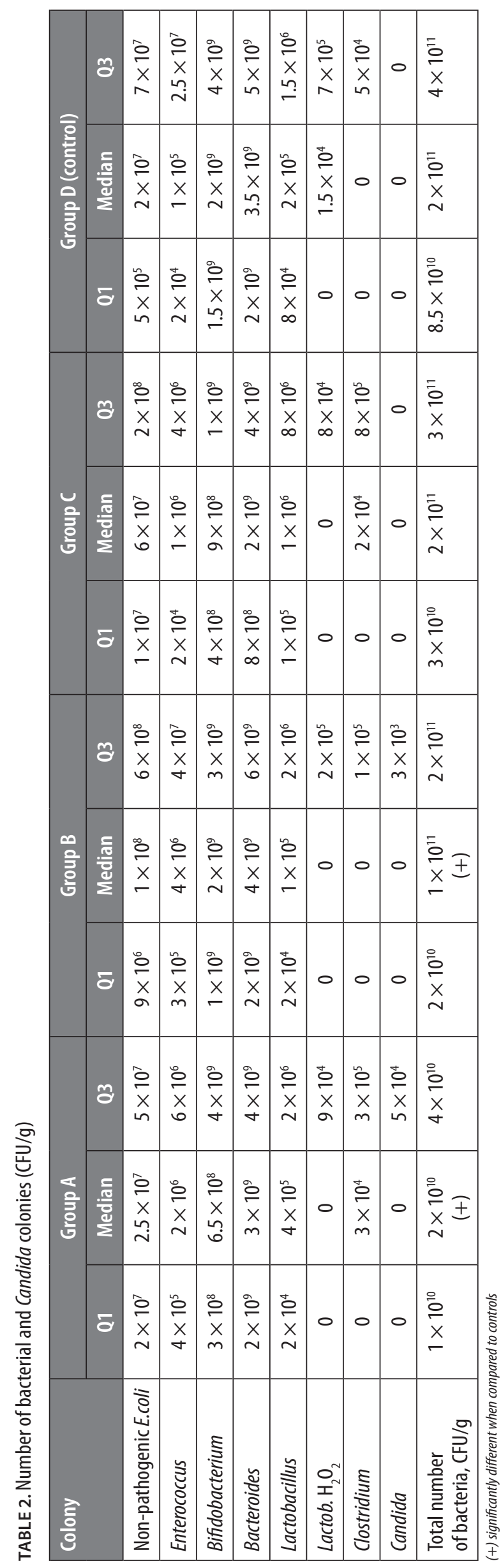

statistically significant difference regarding this variable between groups B and C $(p=0.5)$.

\section{Detailed analysis of bacterial colonies}

CYC-treated children showed a significantly lower number of Bifidobacterium colonies when compared with controls $(p=0.01)$, and also when compared with the GCS-only group $(p=0.04)$.

The number of Bacteroides sp., Lactobacillus, and $\mathrm{H}_{2} \mathrm{O}_{2}$ producing Lactobacillus sp. CFU in groups A, B, and $\mathrm{C}$ was not significantly different when compared with the controls. Likewise, the amounts of CFU of E. coli and Enterococcus sp. showed no significant differences between any of the study groups. Similar results were obtained regarding the Clostridium sp. content.

In the analysis of proteolytic bacteria and molds, we found no significant differences between children with INS and healthy children. However, there was a tendency for overgrowth of other proteolytic bacteria in the CsA group when compared with the controls $(p=0.07)$, with a similar tendency observed in the GCS group $(p=0.06)$. CFUs of E. coli Biovare, Proteus sp., and Pseudomonas sp. were comparable.

\section{Fungi}

Group (A) subjects (CsA) had a significantly higher amount of Candida sp. colonies when compared with the controls ( $p=0.01$ ) (Table 2, Fig. 2). Groups B and C did not show a significant difference when compared with the control group.

\section{CLINICAL SYMPTOMS ANALYSIS AND LABORATORY TESTS}

After analysing the clinical questionnaires, we found that all three groups of children with INS reported symptoms related to gastrointestinal tract, skin disorders, mood changes and anxiety, and tendency to nervous-

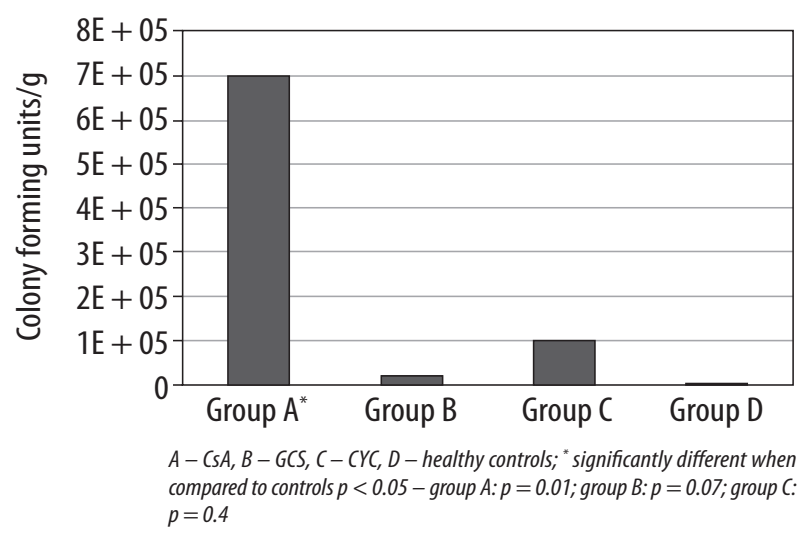

FIGURE 2. Maximum number of Candida sp. growth in the study groups 
TABLE 3. Percentage of children with proteolytic bacterial overgrowth

\begin{tabular}{|l|c|c|c|c|c|}
\hline \multicolumn{2}{|l|}{ Proteolytic bacteria and mold } & Group A (CSA) & Group B (GCS) & Group C (CYC) & Group D (control) \\
\cline { 2 - 6 } & $n=18(\%)$ & $n=17(\%)$ & $n=9(\%)$ & $n=20(\%)$ \\
\hline \multirow{2}{*}{ E. coli Biovare } & Normal growth & $16(89)$ & $13(76.5)$ & $8(89)$ & $19(95)$ \\
\cline { 2 - 6 } & Overgrowth & $2(11)$ & $4(23.5)$ & $1(11)$ & $1(5)$ \\
\hline Proteus sp. & Normal growth & $17(94.4)$ & $17(100)$ & $9(100)$ & $20(100)$ \\
\cline { 2 - 6 } & Overgrowth & $1(5.6)$ & 0 & 0 & 0 \\
\hline Pseudomonas sp. & Normal growth & $18(100)$ & $17(100)$ & $9(100)$ & $18(90)$ \\
\cline { 2 - 6 } & Overgrowth & 0 & 0 & 0 & $2(10)$ \\
\hline \multirow{2}{*}{ Other proteolytic bacteria } & Normal growth & $13(72.2)$ & $12(70.6)$ & $8(89)$ & $19(95)$ \\
\cline { 2 - 6 } & Overgrowth & $5(27.8)$ & $5(29.4)$ & $1(11)$ & $1(5)$ \\
\hline \multirow{2}{*}{ Molds } & Normal growth & $16(88.9)$ & $13(76.5)$ & $8(88)$ & $20(100)$ \\
\cline { 2 - 6 } & Overgrowth & $1(5.6)$ & $4(23.5)$ & $1(11)$ & 0 \\
\hline
\end{tabular}

CSA - cyklosporine A, GCS - glicocorticosteroids, CYC - cyclophosphamide; normal growth $<10^{4}$, overgrowth $>10^{4}$ CFU of microbiota per 1 gram of stool

TABLE 4. Clinical complaints in the idiopathic nephritic syndrome children as reported by their parents

\begin{tabular}{|l|c|c|c|c|c|c|c|}
\hline \multicolumn{2}{|c|}{ Symptom } & \multicolumn{2}{c|}{ Group A (CSA) } & \multicolumn{2}{c|}{ Group B (GCS) } & \multicolumn{2}{c|}{ Group C (CYC) } \\
\cline { 3 - 9 } & & $n=18$ & $\%$ & $n=17$ & $\%$ & $n=9$ & $\%$ \\
\hline \multirow{3}{*}{ Gastrointestinal symptoms } & Present & 12 & 66.7 & 11 & 64.7 & 6 & 66.7 \\
\cline { 2 - 9 } & Absent & 6 & 33.3 & 6 & 35.3 & 3 & 33.3 \\
\hline \multirow{3}{*}{ Mood disorders and anxiety } & Present & 11 & 61.1 & 8 & 47.1 & 4 & 44.4 \\
\cline { 2 - 9 } & Absent & 7 & 38.9 & 9 & 52.9 & 5 & 55.6 \\
\hline \multirow{3}{*}{ Skin problems } & Present & 12 & 66.7 & 9 & 52.9 & 3 & 33.3 \\
\cline { 2 - 9 } & Absent & 6 & 33.3 & 8 & 47.1 & 6 & 66.7 \\
\hline \multirow{2}{*}{ Tendency to nervousness } & Present & 15 & 83.3 & 9 & 52.9 & 6 & 66.7 \\
\cline { 2 - 9 } & Absent & 3 & 16.7 & 8 & 47.1 & 3 & 33.3 \\
\hline
\end{tabular}

CSA - cyklosporine A, GCS - glicocorticosteroids, CYC - cyclophosphamide; no statistically significant differences in these symptoms across the groups ( $p>0.05)$

ness. No significant differences were observed between different protocols of immunosuppression. Almost 67\% of children treated with CsA complained of gastrointestinal symptoms (i.e. constipation, flatulence, dyspepsia, abdominal pain, compulsive hunger or irritability), with similar percentages of children in groups B (64.7\%) and C $(66.7 \%)$ suffering from such symptoms. Mood disorders and anxiety were reported by $60 \%$ of group A patients, and almost half of the patients in groups B and C. Skin problems appeared in about two-thirds, half, and third of the children in groups $\mathrm{A}, \mathrm{B}$, and $\mathrm{C}$ respectively. $83 \%$ of group A children showed tendencies to nervousness, compared to $53 \%$ in group B, and $67 \%$ in group C (Table 4 ). There were no statistically significant differences in these symptoms across all three INS groups $(p>0.05)$.

Selected biochemical parameters were analysed in the INS groups. Some children in group B had elevated levels of WBCs. Cholesterol levels were also elevated in some children from all three groups. The rest of the laboratory test values were within the laboratory norms (Table 5). All patients were in remission, and no acute kidney injury was present during the analysis.

\section{DISCUSSION}

The main finding of our study is that immunosuppressive therapy in patients with INS may induce unfavourable changes in gut microbiota. Different protocols had a slightly different influence on specific microbiota profile. We postulate that this observation might be important for patients from a clinical point of view.

Microorganisms inhabiting the human gastrointestinal tract are in a close symbiotic relation with the human organism. Microbiota perform a variety of functions necessary to maintain homeostasis [2, 5]. Gut microbiota affect the immune system by creating an acidic profile, hydrogen peroxide, antibiotic-like substances, bacteriocins, function peptides, proteases, and nutrients. They also play a role in the local immune system by stimulating GALT to produce sIgA. Some authors reported a favourable effect of the digestive tract physiological flora on carcinogenesis $[2,5,10]$. There is definitely an interdependency between intestinal microorganisms and the immune system. Gut bacteria stimulate development and proper function of immune system components, where- 
TABLE 5. Selected clinical and biochemical parameters in the study groups

\begin{tabular}{|c|c|c|c|}
\hline Parameter & Group A & Group B & Group C \\
\hline Demographics & $\begin{array}{l}18 \text { Children: } 7 \mathrm{~F} ; 11 \mathrm{M} \\
\text { Age: } 2-14 \text { years }\end{array}$ & $\begin{array}{c}17 \text { Children: } 8 \text { F; } 9 \text { M } \\
\text { Age: 2-17 years }\end{array}$ & $\begin{array}{c}9 \text { Children: } 3 \mathrm{~F} ; 6 \mathrm{M} \\
\text { Age: } 3-12 \text { years }\end{array}$ \\
\hline Course of the disease & $\begin{array}{l}\text { Steroid-dependent (16) } \\
\text { Steroid-resistant (2) }\end{array}$ & Steroid-sensitive & Steroid-dependent \\
\hline Number of relapses & $>3$ & $<2$ & $>3$ \\
\hline CsA dose $(\mathrm{mg} / \mathrm{kg} / 24 \mathrm{~h})$ & 3.7 & - & - \\
\hline $\mathrm{GCS}$ dose $\left(\mathrm{mg} / \mathrm{m}^{2} / 48 \mathrm{~h}\right)$ & 10-15 (only 9 children) & $30-40$ & $30-40$ \\
\hline CYC dose (mg/kg/24 h) & - & - & 2.5 \\
\hline Haemoglobin (mg/dl) & $12.9(11-15.1)$ & $13.9(11.7-17.1)$ & $13.35(12-14.2)$ \\
\hline Haematocrit (\%) & $38(33-43.5)$ & $40.3(35.6-45.8)$ & $39.9(34-47)$ \\
\hline Creatinine $(\mathrm{mg} / \mathrm{dl})$ & $0.46(0.15-0.67)$ & $0.43(0.2-0.78)$ & $0.37(0.32-0.46)$ \\
\hline Urea (mg/dl) & $24(13-43)$ & $28(18-35)$ & $29(22-36)$ \\
\hline Uric acid (mg/dl) & $4.7(3-7.4)$ & $3.5(2.9-8.3)$ & $3.9(3.8-4)$ \\
\hline Cholesterol (mg/dl) & $186(159-310)$ & $198(134-261)$ & $202(158.4-247)$ \\
\hline Thrombocytes $\left(\mathrm{mm}^{-3}\right)$ & $381,000(262,000-515,000)$ & $380,500(226,000-458,000)$ & $332,000(208,000-396,000)$ \\
\hline WBCs $\left(\mathrm{mm}^{-3}\right)$ & $7,290(5,330-1,2350)$ & $10,140(6,280-20,000)$ & $7,140(4,840-7,470)$ \\
\hline
\end{tabular}

CSA - cyklosporine A, GCS - glicocorticosteroids, CYC - cyclophosphamide; data is given as the median, quartile values are given in brackets

as the immune system protects against translocation of bacteria to the bloodstream, where these organisms may cause disease $[6,11]$.

The relationship between intestinal microflora and GALT was proven in animal studies. Animals deprived of microbiota in a sterile environment had undeveloped GALT and only single B lymphocytes $[12,13]$. Normal development of GALT was restored after colonization by physiological microflora. Specific intestinal microflora stimulated production of natural antibodies which are part of non-specific immunity. Probiotics showed the ability to recover intercellular tight junctions disrupted by pro-inflammatory cytokines (TNF- $\alpha$, INF-g) $[12,13]$.

In addition to their immunological effects on the host organism, gut microbiota also play critical roles in certain metabolic processes. Intestinal microorganisms influence host metabolic function by producing vitamins $\mathrm{B}_{1}, \mathrm{~B}_{2}, \mathrm{~B}_{12}$, and $\mathrm{K}$, which are absorbed from the gastrointestinal tract. Microbiota are involved in host lipid metabolism by converting cholesterol to coprostanol, degrading bile acids and converting bilirubin to urobilinogen. Changes in microbiota may interfere with the metabolic pathways of these substances leading to long-term clinical consequences.

Changes in intestinal flora composition depend on environmental factors and acquired diseases. In acute diseases, microbiological balance is restored shortly after detrimental factors subside. In chronic diseases, the recovery of proper intestinal flora is more difficult. Adverse effects of antibiotics and selected immunosuppressive drugs on intestinal flora were observed in animal models [10, 14]. Negative consequences of CYC, CsA, and GCS administration on the microbiota have been proven in previous studies on mice $[1,15]$. In our study, we confirmed an imbalance of microbiota induced by immunosuppressive drugs. A diminished total number of bacterial colonies was observed in CsA and GCS-only-treated subjects.

There are few studies examining disturbances of intestinal flora during immunosuppressive treatment, and those available are comprised of case reports and animal model studies [16, 17]. Kaur et al. showed that CsA disturbed intestinal microbiota in mouse model increased the risk of Clostridium difficile (proteolytic bacteria) colonization of the gastrointestinal tract [17]. An increase in Clostridium difficile occurred with diminished protection of commensal bacteria with a particular decrease in Bifidobacterium colonies [18]. In our study, we assessed Clostridium sp. growth and reported no significant increase in the Clostridium sp. colonies in any of the groups.

Gut colonization with Candida is a common complication following any antibiotic therapy. In our study the CsA-treated group showed significant increase in Candi$d a$ colonies, without antibiotic intake. It might be related to the significant decrease in total number of protective bacteria colonies, as reported above.

In children treated with CYC, there was a decrease in the number of Bifidobacterium colonies. However, the low number of patients in this group might have influenced this observation. On the other hand, the CYC therapy protocol is shorter than the one for CsA, thus the changes might be less prominent over time. Samonis et al. reported increased gastrointestinal tract colonization by yeasts in mice on CYC with neutropenia [14]. Shenderov et al. found a negative influence of CYC on gut microbiota in rats [19]. Disturbances in intestinal flora were found only 
when neutropenia occurred. Administering probiotics to re-establish normal gut microbiota resulted in resolution of neutropenia, thus the authors postulated that the severe disturbances of intestinal flora by CYC use might be related to neutropenia [19].

Our study did not confirm a significant increase in proteolytic bacterial colonies, although groups A and $\mathrm{B}$ showed overgrowth tendencies regarding in some proteolytic bacteria as we have mentioned in the results section. These bacteria may increase fermentation processes in the gastrointestinal tract and lead to inflammation $[18,20]$. The GCS influence on microbiota has been described in previous studies. GCS may trigger inflammation of gastric mucosa, which affects the digestion process. Treatment with GCS increases the risk of carbohydrate metabolism disturbances. It may be related to hunger attacks and craving for sweets reported in our questionnaires. Siew et al. investigated the influence of GCS on intestinal flora in the course of nonspecific enteritis. In Crohn's disease they found a decrease in Bacteroides and an increase in invasive $E$. coli colonies. In ulcerative colitis, a low number of Bifidobacterium colonies was observed [21]. On the other hand, the authors reported that GCS administered together with probiotics may improve microbiota profile by decreasing intestinal mucosa inflammatory status. Microbiota status, $\mathrm{pH}$ in the colon, GCS dosage and method of administration were factors influencing effectiveness of the therapy of colitis $[14,22,23]$. Siew et al. also found that GCS together with probiotics at a dose of 3.6 billion CFU, promoted synthesis of anti-inflammatory cytokines (IL-10), inhibited synthesis of pro-inflammatory cytokines (IL-6, IL-12p40) and TLR expression by intestinal dendritic cells [21]. There are no such data in children with INS.

GCS may also disturb gut microflora by increasing susceptibility to mycosis colonization. The tendency to increase in yeast-like fungi in steroid-treated patients may be due to a decrease in the total number of bacterial colonies. This was confirmed in a mouse model [15]. We did not confirm this hypothesis in vivo in children.

Tsuji et al. looked at the relationship between gut microbiota dysbiosis and INS from a different perspective. They examined relapsing INS patients before treatment, and found out that these patients showed gut dysbiosis along with a reduced count of circulatory Tregs [7]. We did not measure the circulatory Treg count, and we compared different treatment protocols in patients during remission, so the two studies cannot be compared to full extent. However, looking at the results from both studies, we suppose that the changes in the gut microbiota can be attributed to the immunosuppressive treatment, as well as the course of the disease itself.

We think that a future study that can analyse a wider variety of INS patient groups (e.g. INS patients on immunosuppressive therapy, INS patients not receiving immunosuppressants, and healthy controls) would be extremely valuable, as such a study would have the potential to show to what extent each factor (the disease itself vs. the immunosuppressive therapy) affects the gut microbiota in those children.

One of the limiting factors to our study was collecting eligible patients. The population of children with INS consists mostly of patients with steroid sensitive, frequently relapsing clinical course. The relapses are usually triggered by upper respiratory tract infections with frequent antibiotic use. Due to this fact, collection of antibiotic-naive patients (to exclude their influence) was a challenge. Thus, restricted inclusion criteria led to a rather low number of participants in our study groups. Moreover, the smaller pool of patients restricted the ability to collect enough subjects receiving only one drug. Thus, half of the children in the CsA group were also receiving GCS, while all the CYC group children received GCS as well. On the other hand, INS children may have disturbances in microbiota because of more frequent antibiotic usage. Long-term antibiotic usage has proven its influence, but there are conflicting data how long the influence is sustained. In our study we relied on other author observations and suggestions from lab test manufacturers to exclude possible changes induced by recent antibiotic administration.

The method of microbiota assessment was based on non-invasive stool testing in order to avoid unnecessary harm to the immunosuppressed patients. However, it gave results of the average composition of bacterial flora, without distinction between different parts of the gastrointestinal tract $[6,11]$.

Despite these limitations, our study has its own robust aspects. All patients were in remission during the analyses, with no acute kidney injury and without any chronic or acute intestinal disease either. No antibiotics or probiotics were used by any of the subjects within two months prior to the study.

\section{CONCLUSIONS}

In conclusion, our study showed that immunosuppression used in childhood INS had unfavourable influence on the intestinal microbiota. The most severe changes were detected in the CsA-treated subjects as they had the lowest total number of bacterial colonies, and the largest growth of Candida sp. colonies. Total number of bacterial colonies was significantly diminished in the GCS-only group as well, while CYC-treated subjects had the lowest number of Bifidobacterium colonies. Clinical gastrointestinal symptoms were not significantly attributed to a specific immunosuppressive agent or to the degree of dysbiosis.

\section{ACKNOWLEDGEMENTS}

The study was partially funded by the Polish Mother's Memorial Hospital Research Institute's funds for research grants. 


\section{DISCLOSURE}

The authors declare no conflict of interest.

\section{REFERENCES}

1. Kinsman OS, Pitblado K. Candida Albicans gastrointestinal colonization and invasion in the mouse: Effect of antibacterial dosing, antifungal therapy and immunosuppression. Mycoses 1989; 32: 664-674.

2. Borriello SP. The normal flora of the intestinal tract. In: Gut Ecology, Hart AL, Stagg AJ, Graffner H, et al. (eds.). Martin Dunitz Ltd., London 2002: 3-12.

3. Hooper LV, Gordon JI. Commensal host-bacterial relationships in the gut. Science 2001; 291: 1115-1118.

4. Hwang JS, Im CR, Im SH. Immune disorders and its correlation with gut microbiome. Immune Netw 2012; 12: 129-138.

5. Wrong OM. Bacterial metabolism of protein and endogenous nitrogen compounds. In: Rowland IR eds. Role of gut flora in toxicity and cancer. Academic Press, New York 1988: 227-262.

6. Kau AL, Ahern PP, Griffin NW, et al. Human nutrition, the gut microbiome, and immune system: envisioning the future. Nature 2011; 474: 327-336.

7. Tsuji S, Suruda C, Hashiyada M, et al. Gut microbiota dysbiosis in children with relapsing idiopathic nephritic syndrome. Am J Nephrol 2018; 47: 164-170.

8. Primary nephrotic syndrome in children: clinical significance of histopathologic variants of minimal change and of diffuse mesangial hypercellularity. A report of the International Study of Kidney Disease in Children. Kidney Int 1981; 20: 765-771.

9. Enck P, Zimmermann K, Rusch K, et al. The effects of maturation on the colonic microflora in infancy and childhood. Gastroenterol Res Pract 2009; 2009: 752401.

10. Si JM1, Yu YC, Fan YJ, Chen SJ. Intestinal microecology and quality of life in irritable bowel syndrome patients. World J Gastroenterol 2004; 10: 1802-1805.

11. Sharma R, Young C, Mshvidobadze M, et al. Mikroflora jelit: czy ma znaczenie w chorobach u noworodków? [Intestinal microflora: is it important in neonatal diesease?]. Medycyna po Dyplomie 2010; 14: 70-86.

12. Cunningham-Rundles S, Ahrne S, Bengmark S, et al. Probiotics and immune response. Am J Gastroenterol 2000; 95 (Suppl 1): S22-25.

13. Isolauri E, Sutas Y, Kankaanpaa P, et al. Probiotics effects on immunity. Am J Clin Nutr 2001; 73: 444-450.

14. Samonis G, Karyotakis NC, Anaissie EJ, et al. Effects of cyclophosphamide and ceftriaxone on gastrointestinal colonization of mice by Candida albicans. Antimicrob Agents and Chemother 1996; 40: 2221-2223.

15. Koh AY, Köhler JR, Coggshall KT, et al. Mucosal damage and neutropenia are required for Candida albicans dissemination. PLoS Pathog 2008: 4: e35.

16. Bowen JRC, Sahi S. Cyclosporine induced colitis. Br Med J 1993; 307: 484.

17. Kaur S, Vaishnavi C, Ray P, et al. Effect of biotherapeutics on cyclosporine-induced Clostridium difficile infection in mice. J Gastroenterol Hepatol 2010; 25: 832-838.

18. Bast A, Chandler RF, Choy PC, et al. Botanical health products, positioning and requirements for effective and safe use. Environ Toxicol Pharmacol 2002; 12: 195-211.

19. Shenderov BA, Glukhova EV, Viadro MM. Microbial ecology of laboratory animals as a model in evaluation of the immunomodulating and antimicrobial agents. Antibiot Khimioter 1992; 37: 39-43.
20. Morgan MY, Blei A, Grüngreiff K, et al. The treatment of hepatic encephalopathy. Metab Brain Dis 2007; 22: 389-405.

21. Siew C, Plamondon S, Kamm MA, et al. Immunosuppressive effects via human intestinal dendritic cells of probiotic bacteria and steroids in the treatment of acute ulcerative colitis. Inflam Bowel Dis 2010; 16: 1286-1298.

22. Friend DR. Review article: issues in oral administration of locally acting glucocorticosteroids for treatment of inflammatory bowel disease. Aliment Pharmacol Ther 1998; 12: 591-603.

23. Tlaskalova-Hogenova H, Stepankova R, Hudcovic T, et al. Commensal bacteria (normal microflora), mucosal immunity and chronic inflammatory and autoimmune diseases. Immunol Lett 2004; 93: 97-108 